\title{
Differential response to frequency-dependent interactions: an experimental test using genotypes of an invasive grass
}

\author{
Alexandra Collins $\cdot$ E. M. Hart $\cdot$ J. Molofsky
}

Received: 13 June 2009/Accepted: 30 June 2010/Published online: 21 July 2010

(C) Springer-Verlag 2010

\begin{abstract}
Positive feedbacks have been suggested as a means for non-indigenous species to successfully invade novel environments. Frequency-dependent feedbacks refer to a species performance being dependent on its local abundance in the population; however, frequency dependence is often described as a monolithic trait of a species rather than examining the variation in response for individual genotypes and fitness traits. Here, we investigate frequency-dependent outcomes for individual genotypes and fitness-related traits for the invasive grass Phalaris arundinacea. We tested for competition-mediated frequency dependence by establishing hexagonal arrays with the center target plant surrounded by either same, different or no genotype neighbors to determine how changing the small-scale frequency neighborhood-influenced invasion success. We used a Bayesian ANOVA approach which allowed us to easily accommodate our non-normal dataset and found that same neighbor plots had greater biomass production than different neighbor plots. Target plants also had greater stem height and aboveground biomass when surrounded by same genotype neighbors. A greenhouse
\end{abstract}

Communicated by Melinda Smith.

A. Collins - J. Molofsky

Department of Plant Biology, University of Vermont,

109 Carrigan Drive, Burlington, VT 05405, USA

E. M. Hart

Department of Biology, University of Vermont,

109 Carrigan Drive, Burlington, VT 05405, USA

Present Address:

A. Collins $(\bowtie)$

Ecology and Evolution Department, University of Fribourg,

Chemin du musée 10, 1700 Fribourg, Switzerland

e-mail: robin.collins@unifr.ch experiment did not support the hypothesis that increased mycorrhizal associations were the cause of positive frequency dependence. We devised a frequency-dependent metric to quantify the extent of fitness-related differences for individual genotypes and found that individual genotypes showed a range of both positive and negative responses to different frequency treatments; however, only positive responses were statistically significant. The smallscale genotypic neighborhood had no effect for the fitnessrelated traits of leaf number, belowground biomass and total biomass. We demonstrate that individual invasive genotypes respond differently to changing frequency neighborhoods and that growth responses do not respond with the same direction and magnitude. A range of frequency-dependent responses may allow genotypes to invade a wide range of environments.

Keywords Frequency dependence . Intraspecific feedbacks · Mycorrhizae ·

Phalaris arundinacea $\cdot$ Genotype $\cdot$ Invasive

\section{Introduction}

Understanding how non-indigenous species are able to invade and take over native communities has become a central question in ecology. Feedbacks, or the sequence of interactions that determine either a positive or negative effect on a system, have been recognized as an important factor contributing to successful plant invasions due to plant-soil feedbacks (Callaway et al. 2004; Farrer and Goldberg 2009; Klironomos 2002; Laungani and Knops 2009; Nijjer et al. 2007; Reinhart and Callaway 2004; Reinhart et al. 2003; and see reviews by Kulmatiski et al. 2008; Reinhart and Callaway 2006; Wolfe and Klironomos 
2005), fire-suppression feedbacks (Stevens and Beckage 2009), feedbacks with pollinators (Agren 1996) and/or competition-mediated feedbacks among species (Harpole and Suding 2007). Frequency-dependent feedbacks refer to a species performance being dependent on its local abundance in the population and, in general, negative frequency-dependent feedbacks are predicted to maintain species diversity (Bever 2003; Molofsky et al. 2002) while positive frequency-dependent feedbacks are predicted to maintain species uniformity (Bever 2003; Silvertown and Charlesworth 2001; but see Molofsky et al. 2001 for an exception). Thus, invasion potential may be increased if plants exhibit positive frequency-dependent feedbacks (Klironomos 2002; Bever 2003). Yet, most studies have focused only at the species level and our understanding of how intraspecific frequency-dependent interactions influence invasion success remains limited. A fundamental level of variation occurs at the genotype level (Aarssen and Turkington 1983; Antonovics 1976; Harper 1977), and our understanding of how intraspecific frequency-dependent feedback mechanisms increase invasion success may further our ability to predict invasion risk.

Competition-mediated intraspecific frequency-dependent feedbacks driven by neighbors have been recognized for plants (Kelley and Clay 1987; Turkington 1979) and have been shown to influence plant fitness at small spatial scales (Aarssen and Turkington 1985; Antonovics and Ellstrand 1984; Crutsinger et al. 2008). Yet, frequency dependence is generally described as a monolithic trait of all genotypes and all traits (Antonovics and Ellstrand 1984), when in reality genotypes may have considerable variability in their frequency-dependent response. For example, Ronsheim (1996) found that different genotypes of Allium vineale responded differently to neighbor identity. Two out of five genotypes showed evidence for positive frequency dependence while others showed no effect of neighbor identity. Similarly, models investigating frequency dependence often assume that the magnitude and direction of frequency dependence is identical for all individuals (Molofsky and Bever 2002; Molofsky et al. 2001, 2002; but see Eppstein et al. 2006 for an exception). In a two-species model, both the strength and sign of frequency dependence (i.e., whether positive or negative) influenced the patterns of coexistence (Eppstein et al. 2006). Furthermore, the small-scale genotypic diversity of plant populations has been shown to have extended consequences for the diversity of associated herbivores (Crutsinger et al. 2006; Johnson and Agrawal 2005, 2007; Johnson et al. 2006) and for the ability of invasive species to establish (Crutsinger et al. 2008), yet to our knowledge very few studies have examined how individual genotype interactions influence invasive plant performance (but see Vellend et al. 2010).
In addition to understanding the variability in invasive genotype response, determining the mechanisms driving these changes will also better inform our ability to predict genotype trajectories and invasive spread. For plants, negative frequency dependence has been shown to occur through competition and niche partitioning (Antonovics and Kareiva 1988), parasitism (May and Anderson 1983) or mutualism through an asymmetry in benefits to mutualists (Bever 1999), while positive frequency dependence has been shown to occur by preferential predation on rare individuals (Futuyma and Wasserman 1980), host specificity amongst mutualists (Bever 1999) or increased mycorrhizae hyphal connections amongst like genotypes (Ronsheim and Anderson 2001).

Here, we examine the variability of individual genotype response to neighbor identity for multiple growth measures using genotypes of the invasive wetland grass Phalaris arundinacea. Phalaris arundinacea has been shown to have maintained high genotypic diversity in the invasive range (Lavergne and Molofsky 2007) and genotypic diversity is predicted to have its largest ecological effect when the community is dominated by one or a few primary species (Hughes et al. 2008; Whitham et al. 2006). Our objectives were threefold: (1) to determine the extent of positive or negative frequency-dependent interactions by measuring multiple traits for plant growth, (2) to investigate mycorrhizal hyphal association as the mechanism for positive frequency dependence, and (3) to quantify the extent of growth differences under different small-scale frequency neighborhoods for each individual genotype. We use a flexible statistical approach, Bayesian ANOVA (Qian and Shen 2007), to accommodate both normal and nonnormal data. Our work allows for the further understanding of intraspecific frequency-dependent processes and how this may influence invasion success by examining plant performance under different frequency scenarios and the extent to which individual genotypes and growth responses vary in their frequency dependence.

\section{Materials and methods}

\section{Study species}

Phalaris arundinacea (Poaceae), reed canary grass, is a cool season perennial $\mathrm{C}_{3}$ grass that is native to temperate zones of the northern hemisphere and is widely distributed throughout Eurasia (Lavergne and Molofsky 2007). Plants grow between 1 and $2 \mathrm{~m}$ tall with dense panicles giving rise to both fertile and sterile florets (Gleason and Cronquist 1991). $P$. arundinacea has high annual seed set as well as a high rate of outcrossing due to self-sterility (Ostrem 1988), and can also reproduce asexually by producing clonal tillers. 
$P$. arundinacea is naturally mycorrhizal with colonization varying between 3 and $90 \%$ depending on the sampling location (Bauer et al. 2003; Beck-Nielsen and Madsen 2001; Cooke and Lefor 1998; Rickerl et al. 1992). An extensive belowground rhizome network makes $P$. arundinacea a competitively dominant species, and it is currently considered an invasive species in many northeastern states of the USA (Kilbride and Paveglio 1999; Lavergne and Molofsky 2004).

$P$. arundinacea is a good study species for experiments on genotypic differences because individuals can be easily genotyped through allozyme screening and rapidly cloned through repeated vegetative tillering. Previous collections of genotypes from the invasive range of $P$. arundinacea have found that genotypic differences amongst plants translate into differences in physiological and morphological characteristics (Broderson et al. 2008; Morrison and Molofsky 1999). Furthermore, differences in these characteristics result in differences in competitive ability and survival (Morrison and Molofsky 1998).

Frequency dependence experiment

We experimentally manipulated the intraspecific frequency of genotypes using hexagonal arrays with one "target" plant in the center of the array surrounded by six "neighbor" plants. We used a 3-cm planting density because that corresponds to plant distances observed under field conditions (Collins, personal observation). The experiment consisted of three treatments: (1) target plant with no neighbors, (2) target plant surrounded by six same genotype neighbors, and (3) target plant surrounded by six different genotype neighbors, with each of the six neighbor genotypes representing a different genotype. We established the no neighbor treatment as a control to assess the amount of competition target plants experienced when grown with neighbors. Using the neighbor treatments, we tested for competition-mediated frequency dependence where increased performance of the same neighbor treatment provides support for positive frequency dependence (or reduced intragenotypic competition) and increased performance of the different neighbor treatment provides support for negative frequency dependence (or increased intragenotypic competition). We quantified performance by measuring one fitness response (tiller number) and six growth responses (stem height, leaf number, tiller number and aboveground, belowground and total biomass). Tiller number served as a good proxy for asexual fitness and potential for spread as $P$. arundinacea produced no seeds during the course of our 2-year experiment.

We used a total of seven genotypes and each of the seven genotypes served as the target plant for the three treatments and was replicated three times for a total of 63 plots. All plants were pruned at the time of planting to have two green leaves, $10 \mathrm{~cm}$ of stem, $5 \mathrm{~cm}$ of roots, $2 \mathrm{~cm}$ of rhizome, and one rhizome growing tip. Any genotypes that died after 2 weeks were determined to have died from transplant shock and were replaced. No other plants were replaced after 2 weeks.

The experiment was conducted at a wetland site that had pre-existing stands of $P$. arundinacea [Biological Research Complex (BRC) Burlington, Vermont $\left.\left(44^{\circ} 27^{\prime} \mathrm{N}, 73^{\circ} 11^{\prime} \mathrm{W}\right)\right]$.

The seven genotypes were collected from one of three established populations of $P$. arundinacea in Vermont [Shelburne Bay $\left(44^{\circ} 24^{\prime} \mathrm{N}, 73^{\circ} 14^{\prime} \mathrm{W}\right)$, Gavin Hill $\left(44^{\circ} 35^{\prime} \mathrm{N}\right.$, $\left.73^{\circ} 08^{\prime} \mathrm{W}\right)$ and Ethan Allen Homestead $\left(44^{\circ} 30^{\prime} \mathrm{N}\right.$, $\left.\left.73^{\circ} 14^{\prime} \mathrm{W}\right)\right]$. We used allozymes to identify seven unique genotypes (see Lavergne and Molofsky 2007) and vegetatively propagated all genotypes in the greenhouse prior to being planted at the field site. Clonal replicates of each genotype were generated by cutting stems from existing pots and adding rooting hormone to each node along the length of the stem. Stems were then laid in soil-filled flats and covered with soil. New plants were produced from each node within 6-8 weeks under saturated soil conditions and simulated spring temperatures $\left(22-26^{\circ} \mathrm{C}\right.$ day $/ 16-20^{\circ} \mathrm{C}$ night with $12 \mathrm{~h}$ days).

All plots were monitored on a bi-weekly basis for both the 2006 and 2007 field seasons (July-September 2006 and May-September 2007) for survivorship, tiller number and the growth response traits of stem height and leaf number. On 4 September 2007, the above- and belowground biomass for all target and neighbor plants was harvested from each plot. Belowground biomass was washed and aboveand belowground biomass was dried at $60^{\circ} \mathrm{C}$ for $48 \mathrm{~h}$ before being weighed.

\section{Mycorrhizae experiment}

If mycorrhizal associations among like genotypes is the mechanism responsible for positive frequency dependence, than genotypes must have genotype-specific responses to particular mycorrhizal strains (Ronsheim 1996). Here, we test the hypothesis that hyphal networks amongst like genotypes will generate a positive feedback. $P$. arundinacea can be highly mycorrhizal (up to $90 \%$ colonization), and therefore the presence of mycorrhizal fungi is a plausible mechanism affecting frequency dependence. To test for genotype specific mycorrhizal associations, we conducted a greenhouse experiment where we planted target genotypes with and without genotype neighbors in both mycorrhizal and non-mycorrhizal (fungicide-treated) soil. Target genotypes were planted in similar arrays to our frequency dependence field experiment, with either three same genotype neighbors, three different neighbor genotypes or no neighbors. Genotypes were collected from the 
same three populations in Vermont as our frequencydependent experiment and were grown under greenhouse conditions for multiple generations to remove any maternal effects prior to the establishment of the experiment. The experiment consisted of three neighbor treatments, two soil treatments, and four genotypes all replicated six times for a total of 144 pots. Soil for the experiment was collected from the field site where the frequency dependence experiment was planted in August 2008 and mixed 1:1 with perlite to improve soil aeration. Prior to soil removal, existing $P$. arundinacea plants were removed and roots were cleared and stained to confirm the presence of mycorrhizal fungi at our experimental site. We also collected three composite soil samples that were analyzed by the University of Vermont soil testing laboratory for available soil phosphate.

Plant arrays were established in 1-gallon (c. 4.5-L) pots, and humidity and other abiotic factors were regulated and maintained constant throughout the entire experiment. The temperature of the greenhouse was maintained at $23.8^{\circ} \mathrm{C}$ day $/ 15.5^{\circ} \mathrm{C}$ night. We eliminated mycorrhizae by applying the fungicide Topsin-M in solution (70\% a.i.; Cerexagri, Philadelphia, PA, USA) at a rate of $50 \mathrm{mg}$ (active ingredient) $\mathrm{kg}^{-1}$ soil (dry mass). Topsin-M was added as $200-\mathrm{mL}$ aliquots to each pot at the beginning of the experiment and re-applied every 3 weeks. Topsin M: thiophanate-methyl: dimethyl [1,2-(phenylene)-bis(iminocarbonothioyl)] bis (carbamate) is a fungicide with a similar mechanism for fungal suppression as the now banned fungicide, benomyl (methyl 1-[butylcarbamoyl]-2-benzimidazole carbamate), and significantly reduces AMF colonization (Wilson and Williamson 2008).

Genotypes were planted in their treatments on 29 July 2008 and we measured both the target and neighbor plants for the traits of stem height, leaf number and tiller number every 3 weeks. We harvested the experiment 10 October 2008 and separated above- and belowground biomass for each individual genotype in each pot. $P$. arundinacea has distinctive rhizomes making individual genotypes easy to distinguish.

\section{Statistical analysis}

\section{Bayesian model}

We analyzed our data using Bayesian ANOVAs which are sometimes called Bayesian hierarchical linear randomeffects models (Gelman and Hill 2007; Qian and Shen 2007). We chose this approach because it allows us to use a single comprehensive analytical framework for both our normal and non-normal data. These kinds of models can be built with a variety of methods (penalized quasi-likelihood, Laplace approximation) but the Bayesian Markov-Chain Monte-Carlo (MCMC) method is one of the most flexible
(Bolker 2008). We used the statistical package $\mathrm{R}$ (R Development Core Team) in conjunction with WinBUGS (Gilks et al. 1994) to fit the following generalized model.

$Y_{j k l} \sim N\left(\mu_{j k l}, \sigma^{2}\right)$

$\mu_{j k l}=\beta_{0}+\beta_{1 j}+\beta_{2 k}+\beta_{3 l}+\beta_{4 l j}$

In the above generalized formulation, $\beta_{0}$ is the grand mean, $\beta_{1}$ is the effect of neighbor treatment $j, \beta_{2}$ is the effect of study year $k, \beta_{3}$ is the effect of genotype $l$ and $\beta_{4}$ is a term for the interaction between genotype $l$ and neighbor treatment $j$. Each effect term is then modeled as a random normal variable with a mean of 0 and variance $\sigma^{2}$

$\beta_{1 \ldots 4} \sim N\left(0, \sigma_{\beta_{1 \ldots 4}^{2}}^{2}\right)$.

Variance between groups is the estimated variance term in Eq. 2. A second measure of between group variance is known as finite population variance $s^{2}$, which is the standard deviation of each $\beta_{1 \ldots 4}$. When the number of groups is large, $s^{2}$ and $\sigma^{2}$ will converge, otherwise $s^{2}$ is a more accurate estimate of between group variability (Gelman and Hill 2007). Each of the three response variables were fit to the full model (Eq. 1) with some changes made to accommodate the dataset. Mean stem height was fit to the full model (Eq. 1), while aboveground biomass measurements were fit to a model without study year, since measurements were only made in 2007 when the experiment was harvested.

$Y_{j k l} \sim \operatorname{LogNormal}\left(\mu_{j l}, \sigma^{2}\right)$

$\mu_{j k l}=\beta_{0}+\beta_{1 j}+\beta_{2 l}+\beta_{3 l j}$

Tiller number on final harvest was fit to a model that takes into account that tiller number is a Poisson-distributed count variable.

$Y_{j k l} \sim \operatorname{Pois}\left(\mu_{j k l}\right)$

$\mu_{j k l}=\beta_{0}+\beta_{1 j}+\beta_{2 k}+\beta_{3 l}+\beta_{4 l j}$

Each model was fit in WinBUGS called from $\mathrm{R}$ using R2WinBUGS using 3 chains and 50,000 iterations with a 10,000 iteration burn-in period (Sturtz et al. 2005). The significance of an effect was determined by whether a $95 \%$ credible interval bounded zero; if an effect bounded zero it was considered to be not significant.

\section{Frequency dependence for individual genotypes}

We quantified the extent of growth differences for our seven genotypes under both our same and different neighbor treatments. These metrics were calculated using the same three growth measures measured in our Bayesian model: mean stem height, aboveground biomass and final tiller number at harvest. We standardized growth (stem and 
tiller) and biomass measures for treatments by subtracting the mean of the target individuals for genotype $i$ of the same neighbor treatment $\left(T_{i s}\right)$ from the mean of the target individuals for genotype $i$ from the different neighbor treatment $\left(T_{i d}\right)$ and dividing by the mean of the target individual same neighbor treatment (Eq. 5).

$E_{i}=\left(T_{i s}-T_{i d}\right) / T_{i s}$

where, $E_{i}$ represents the extent of the growth differences between the two neighbor treatments for genotype $i$. Here, positive values indicate the extent of positive frequency dependence and negative values indicate the extent of negative frequency dependence. All $E_{i}$ estimates were calculated with a permutation test with 10,000 replicates in R to calculate $P$ values.

\section{Mycorrhizae experiment}

We analyzed the mycorrhizae data using the Bayesian ANOVA models described above, except instead of a year factor, $k$ was the fungicide treatment. Stem height, leaf number, tiller number and biomass measures were our response variables and genotype, neighbor identity and soil type were treated as fixed effects. We also modeled interactions between genotype and fungicide treatment and between neighbor treatment and fungicide treatment. All response variables were modeled with the same error distributions as in the preceding models (e.g., leaf with a Poisson distribution) except for tiller number. We normally would model tiller number as a Poisson variable, but because of poor convergence we modeled it as a normal variable after a square root transformation. We again used WinBUGS and R2WinBUGS with 50,000 replicates with a 10,000 replicate burn-in and three chains. Significance was again assessed by whether or not $95 \%$ credible intervals bounded 0 for a given treatment.

\section{Results}

For all growth responses measured, the no neighbor treatment had consistently greater performance than the neighbor treatments indicating that plants in arrays experienced competition when grown with neighbors; however, this result was only statistically significant for tiller number (Fig. 1c). Comparing only the neighbor treatments, we found that the total plot biomass (including both target and neighbor plants) was significantly greater for same neighbor treatments than different neighbor treatments (Fig. 2). Survivorship of target plants was also greater under the same neighbor treatments than the no neighbor treatments; however, this effect was not statistically significant. For target plants, same and different neighbor treatments
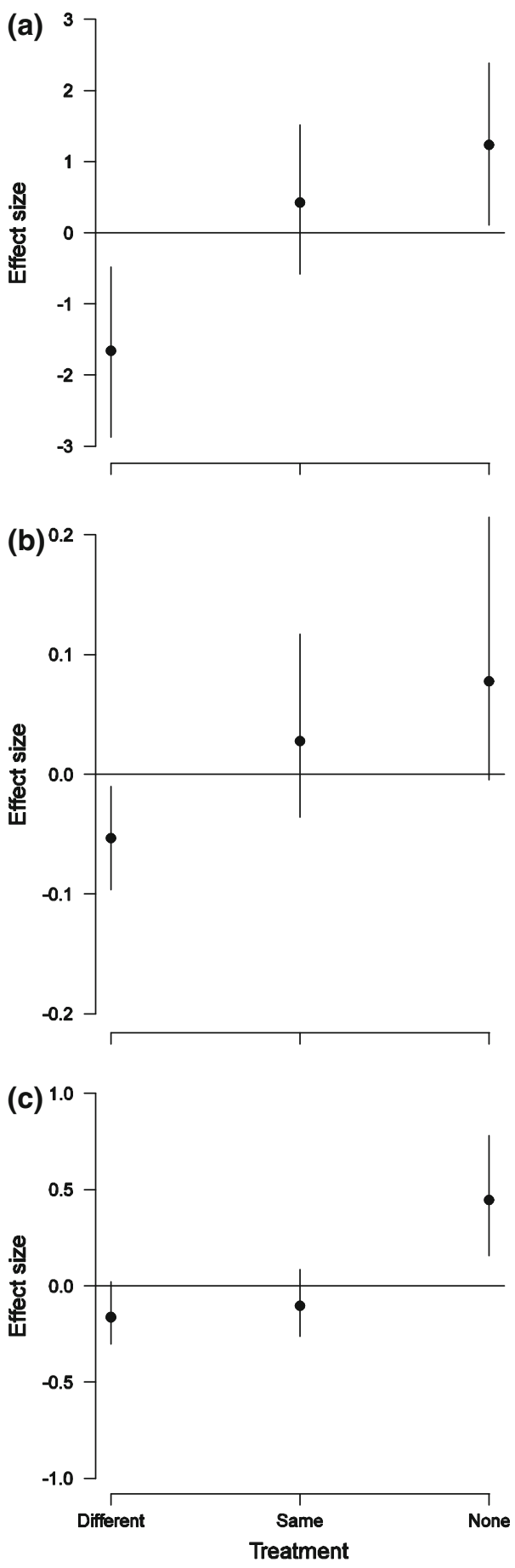

Fig. 1 Estimates of the effect size of neighbor treatment for Phalaris arundinacea on a stem height, $\mathbf{b}$ aboveground biomass and $\mathbf{c}$ tiller number. Same refers to the same neighbor treatment, Different to the different neighbor treatment and None to the no neighbor treatment. Each estimate is the effect relative to the grand mean of the trait. Thin lines represent $95 \%$ credible intervals and the center point is the median of the posterior distribution

showed no neighbor treatment or genotype effect for the traits of leaf number, belowground biomass and total biomass. Here, we focus on the fitness response (tiller number) 


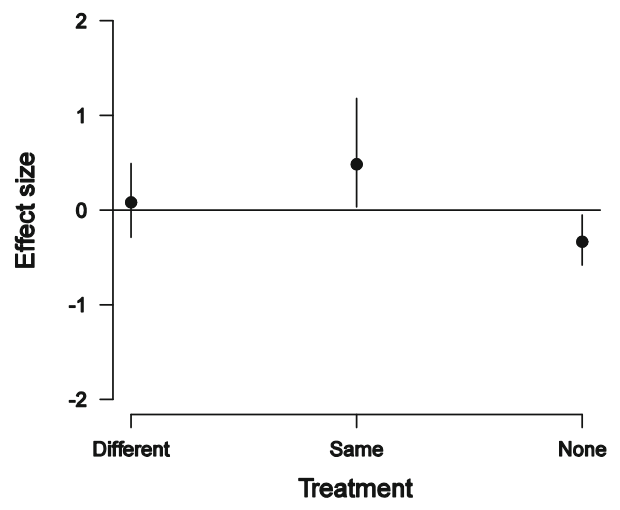

Fig. 2 Estimates of the effect size of neighbor treatment on the total biomass produced by each plot (including target plants and neighbors). Same refers to the same neighbor treatment, Different to the different neighbor treatment and None to the no neighbor treatment. Each estimate is the effect relative to the grand mean of the trait. Thin lines represent $95 \%$ credible intervals and the center point is the median of the posterior distribution

and growth responses (stem height and aboveground biomass) that showed significant genotype and neighbor treatment effects.

Fitness response

The largest variance component for tiller number (excluding the residual variance) was neighbor treatment (Fig. 3c). The presence of neighbors increased competition such that genotypes performed best with no neighbors and there was no difference in performance between same and different neighbor treatments (Fig. 1c). Tiller number was the only response variable measured that showed a significant effect of genotype (Fig. 3c).

\section{Growth responses}

Target plants surrounded by different genotype neighbors had smaller mean stem height, and smaller aboveground biomass than target plants surrounded by same genotype neighbors (Fig. 1a, b). Interestingly, for both stem height and aboveground biomass, there was no significant difference in performance between the same neighbor and no neighbor treatments (Fig. 1a, b) indicating that the presence of same genotype neighbors promoted positive frequency-dependent interactions that may have overcome the negative effects of competition.

Individual genotypes differed in performance under neighbor treatments; however, the effect of neighbor treatment was so large that any effect of genotype was not significant, and for stem height, the inclusion of study year explained more variance than genotype (Fig. 3a).

Aboveground biomass had the greatest neighbor treatment $\times$ genotype interaction indicating that different
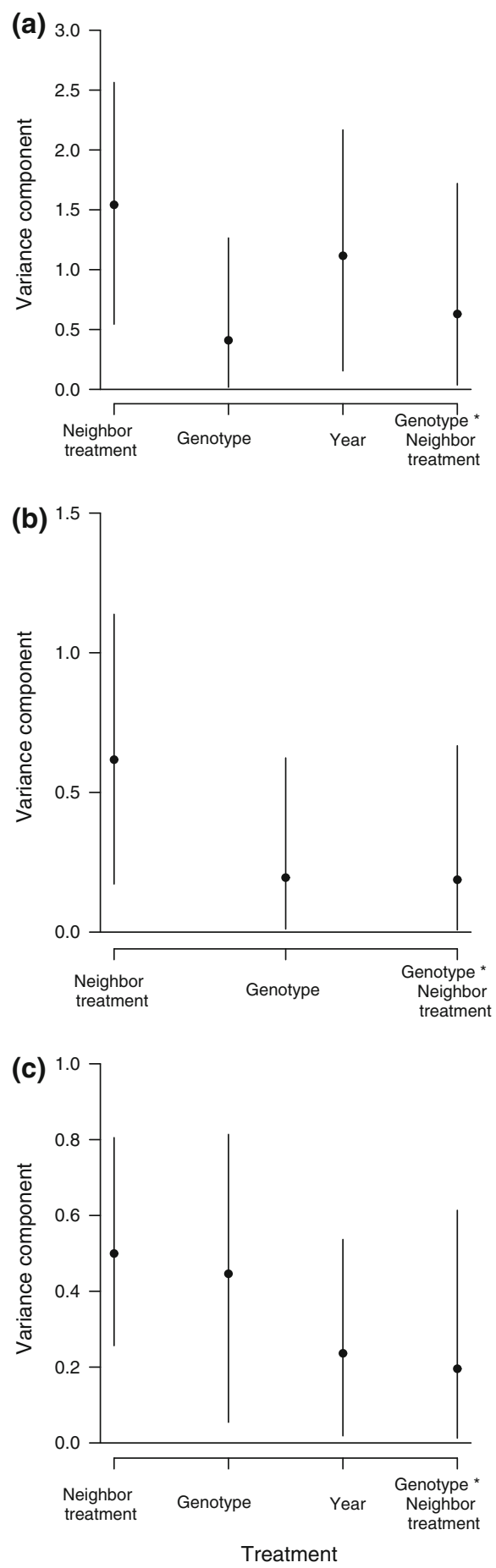

Fig. 3 Estimates of variance components for a stem height, $\mathbf{b}$ aboveground biomass and $\mathbf{c}$ tiller number. Each estimate is the effect relative to the grand mean of the trait. Thin lines represent $95 \%$ credible intervals and the center point is the median of the posterior distribution. Neighbor treatment refers to whether genotypes were planted with same, different or no neighbors, Genotype to the seven genotypes used in the study and Year to the two sampling years of the study. Year is not included for aboveground biomass because the biomass measurements were only collected for the final year of the experiment. The significance of an effect is determined by whether the $95 \%$ credible interval bounds zero; if an effect bounds zero it is not considered significant 

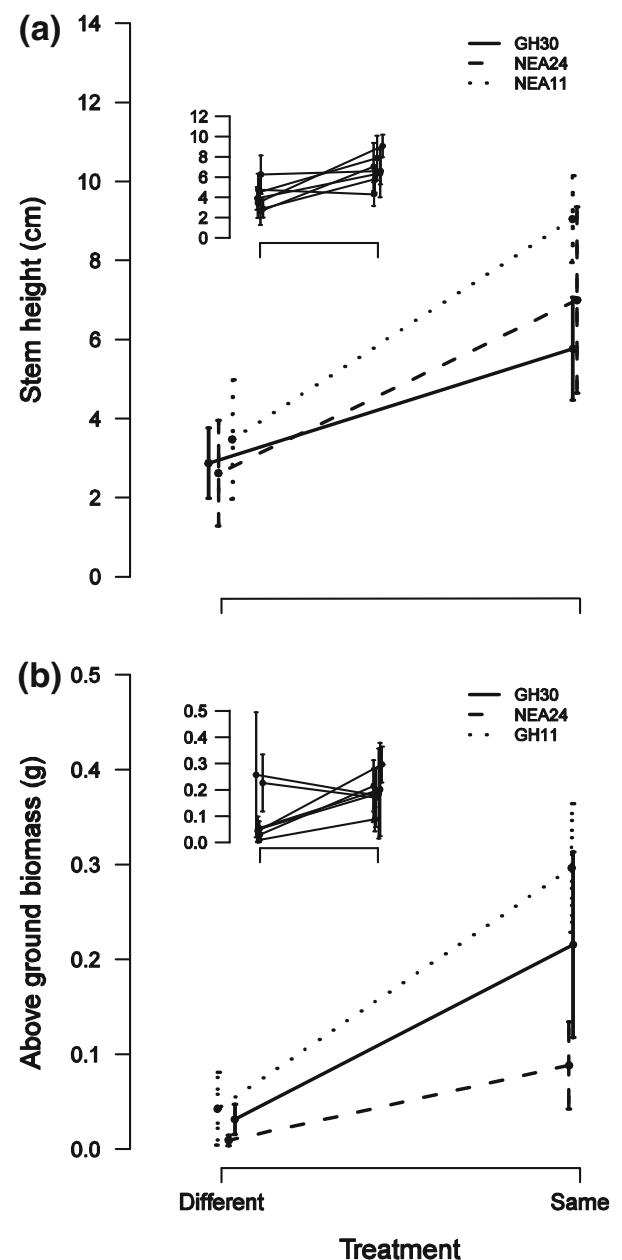

Fig. 4 Interaction plots for $\mathbf{a}$ stem height and $\mathbf{b}$ aboveground biomass with standard error bars. Inset figures shows the response of all seven genotypes and the main figures show only those genotypes with a frequency metric that had a confidence interval that did not bound zero and thus can be considered statistically significant. $G H$ and $N E A$ refer to the population of genotype collection

genotypes responded differently to neighbor frequency. Five genotypes performed better when surrounded by the same neighbor genotypes while two genotypes performed worse (Fig. 4b, inset). Stem height had six genotypes performing better when surrounded with like neighbors (Fig. 4a, inset). As stem height and aboveground biomass were the two traits to show differences in performance between neighbor treatments, and for many genotypes the standard error was large, we examined which genotype effects were significant by quantifying the extent of frequency dependence for individual genotypes.

Frequency-dependent growth for individual genotypes

To exclude the possibility that some genotypes were simply competitively dominant over others, we compared growth responses of genotypes when grown alone and
Table 1 Frequency-dependent metrics for $P$. arundinacea growth measures for seven unique genotypes using a permutation test with 10,000 replicates

\begin{tabular}{lllcc}
\hline & Stem height & $P$ value & $\begin{array}{c}\text { Aboveground } \\
\text { biomass }\end{array}$ & $P$ value \\
\hline GH10 & 0.05 & 0.44 & -0.32 & 0.63 \\
GH11 & 0.42 & 0.06 & $\mathbf{0 . 8 5}$ & $\mathbf{0 . 0 5}$ \\
GH30 & $\mathbf{0 . 5}$ & $\mathbf{0 . 0 5}$ & $\mathbf{0 . 8 9}$ & $\mathbf{0 . 0 4}$ \\
NEA11 & $\mathbf{0 . 6 3}$ & $\mathbf{0 . 0 3}$ & 0.74 & 0.26 \\
NEA24 & $\mathbf{0 . 6 1}$ & $\mathbf{0 . 0 2}$ & $\mathbf{0 . 8 6}$ & $\mathbf{0 . 0 2}$ \\
SB12 & 0.38 & 0.18 & -0.43 & 0.54 \\
SB3 & -0.11 & 0.58 & 0.73 & 0.26 \\
\hline P0sitive
\end{tabular}

Positive effect sizes mean that plants performed better when planted with same genotype neighbors, and negative effect sizes mean that plants performed better when planted with different genotype neighbors. Values in bold are statistically significant $(P \leq 0.05)$

found that there were no significant differences in growth among our seven genotypes. We then examined the interaction effect of the neighbor treatment $\times$ genotype interaction by calculating frequency metrics for all genotypes for the growth responses of stem height and aboveground biomass.

In neighbor treatments, genotypes showed a range of both positive and negative frequency-dependent interactions (Table 1); however, all genotypes that exhibited a negative frequency-dependent response were not significant. Two genotypes, NEA 24 and GH 30, had significant positive frequency-dependent interactions for both stem height and aboveground biomass measures. In total, three genotypes showed significant evidence for positive frequency-dependent interactions for stem height (GH30, NEA11 and NEA24) and three genotypes showed evidence for positive frequency-dependent interactions for aboveground biomass (GH11, GH30 and NEA 24) (Table 1; Fig. 4).

Testing the mechanism driving positive frequency dependence

Plants had greater stem height and aboveground biomass when planted in the fungicide-treated soil (Fig. 5). Stem height had a significant interaction with the fungicide treatment because plants in the no neighbor treatment had a greater effect of the fungicide treatment than neighbor treatments and there was no significant difference between the same and different neighbor treatments. Neighbor treatment had a significant effect for the traits of stem height, tiller number and aboveground biomass. All growth measures were significantly greater under the no neighbor treatment and we found no significant difference between the same and different neighbor treatments. The particular genotype used had a significant effect for tiller number; however, we found no significant interactions (Fig. 5). 

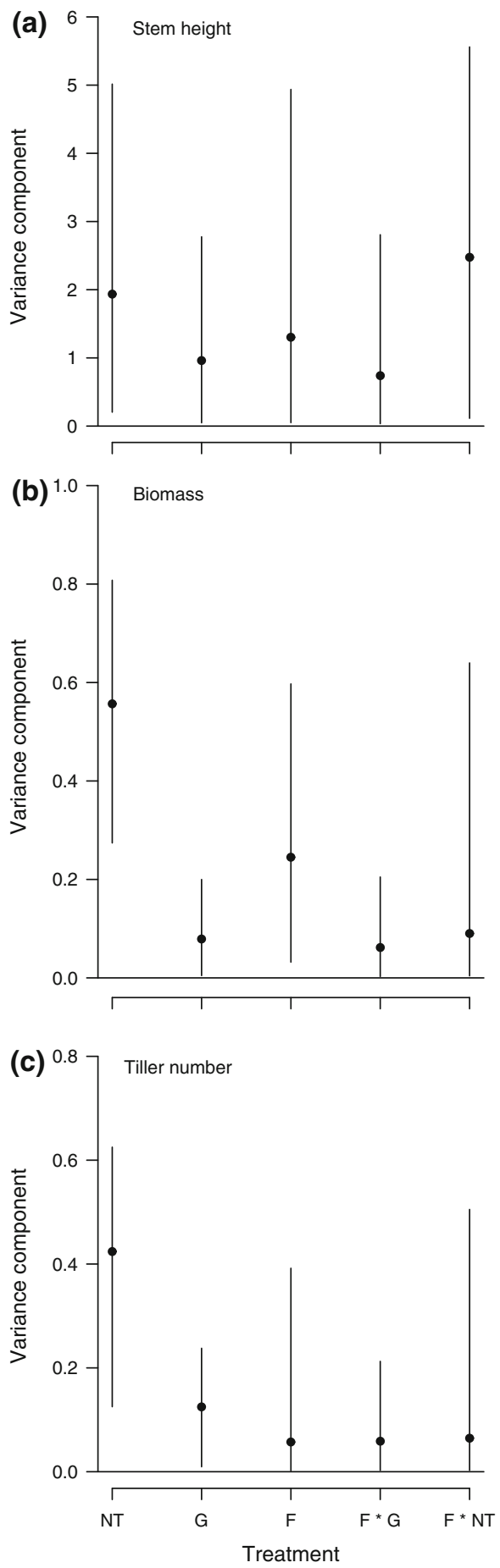

Fig. 5 Estimates of variance components for mycorrhizae experiment for a stem height, $\mathbf{b}$ aboveground biomass and $\mathbf{c}$ tiller number. Each estimate is the effect relative to the grand mean of the trait. Thin lines represent $95 \%$ credible intervals and the center point is the median of the posterior distribution. NT refers to the neighbor treatment and whether genotypes were planted with same, different or no neighbors, $G$ refers to genotype and $F$ to the fungicide treatment and whether or not the soil was treated with the fungicide Topsin-M. The significance of an effect is determined by whether the $95 \%$ credible interval bounds zero; if an effect bounds zero, it is not considered significant
Analysis of soil samples taken from the site of soil collection found that the mean amount of soil phosphorus was $5.65 \mathrm{ppm}$ which is considered optimum in Vermont.

\section{Discussion}

Invasion potential may be increased if plants exhibit positive feedbacks (Klironomos 2002; Bever 2003). Interestingly, here we show that at the genotype level, positive frequency dependence increases invasive plant performance for several, but not all, growth measures. Stem height and aboveground biomass both showed evidence for intraspecific positive frequency dependence. For $P$. arundinacea, positive frequency-dependent interactions that result in taller stem heights may decrease time to seed set and increase spread through greater seed recruitment. There appears to be a threshold stem height of approximately $30 \mathrm{~cm}$ before plants will set seed (Collins, personal observation). In addition, same neighbor and no neighbor treatments had no significant difference in performance for stem height and aboveground biomass. Thus, the presence of same genotype neighbors did not significantly reduce plant performance and may therefore increase invasive spread, particularly for newly invaded sites. Plants grown with same genotype neighbors may have less belowground competition (e.g., sharing resources underground) and may therefore allocate more energy to aboveground biomass and stem growth.

Positive frequency dependence may also help invasive species overcome potential Allee effects (Allee 1931) that can limit invasive spread (Elam et al. 2007). An Allee effect occurs when an individual's performance increases with population size or density (Stephens et al. 1999) and can lead to decreased establishment, longer lag times and slower rates of spread (Taylor and Hastings 2005). For $P$. arundinacea, increased positive frequency-dependent interactions for growth measures such as aboveground biomass may promote the formation of dense monocultures leading to the exclusion of native plants even when propagule pressure is low. We found that genotypes produced greater biomass when planted with same genotype neighbors. Thus, the lack of genotypic variation at new sites of invasion may not limit invasion success. Finding increased productivity in monoculture is in contrast to other studies that have found greater overall productivity in genetically diverse plots (Johnson et al. 2006; Crutsinger et al. 2006). Interestingly, previous studies examining the effect of genotypic diversity have primarily used native species, and we suggest that positive frequency-dependent interactions among invasive genotypes may facilitate invasion into new environments. 
We also found considerable variation in how different growth measures respond to changing the frequency neighborhood. Although most genotypes exhibited greater performance when grown with like neighbors, the extent of positive frequency-dependent interactions varied among genotypes and the growth measures examined. Furthermore, three growth measures (leaf number, belowground biomass and total biomass) showed no evidence for any frequency-dependent interactions. Given the relatively small number of genotypes used in our experiment and the large variability in response, this suggests that even at the invasive patch level there is a considerable range of responses to neighbor identity. A range of positive and negative frequency-dependent growth responses within a patch could ultimately impact established patch structure and influence local genotypic diversity (Eppstein et al. 2006). Negative frequencydependent interactions amongst genotypes could promote genotypic diversity within a patch while the presence of genotypes that have positive frequency-dependent interactions could result in the formation of stable genotype clusters (Molofsky et al. 2001). Determining the extent of negative or positive intraspecific frequency-dependent interactions in established invasive populations will require a more rigorous sampling within a single patch as well as the determination of which specific plant traits are under frequency-dependent selection.

To our knowledge, no other study has quantified the extent of performance differences due to frequencydependent interactions for individual invasive genotypes. What sets our work apart is that, rather than finding no frequency-dependent effect (Bennington and Stratton 1998) or pooling the frequency-dependent effects of all individual genotypes (Antonovics and Ellstrand 1984), we successfully quantify the amount of positive and negative frequency-dependent interactions for different growth measures for each individual genotype used in our study. As an increasing amount of work shows that genotypic diversity greatly influences community structure (Neuhauser et al. 2003; Whitham et al. 2003), quantitative studies such as ours will help to untangle how feedbacks impact genotype coexistence and may in turn clarify patterns we see at higher trophic levels. For example, Booth and Grime (2003) found that increasing genotypic variation of a longterm grassland experiment reduced the rate at which species diversity declined. A follow-up study (Whitlock et al. 2007), examining the role of genetic variation for shaping community composition, found that patterns of abundance in these plant communities could be predicted from knowing the genotypic composition of their component populations. Therefore, knowing how genotypes interact within established invasive patches and how their interaction influences performance could help predict their potential to outcompete native species and for future invasion risk.

We document cases of significant positive frequencydependent interactions for certain invasive genotypes; however, what remains unclear is what mechanisms are driving these increases in plant performance. Empirical studies have proposed several potential mechanisms leading to positive frequency dependence including predation of rare species (Futuyma and Wasserman 1980), host specificity (Bever 1999) and shared mycorrhizal associations (Ronsheim and Anderson 2001). We predicted that mycorrhizal associations may have affected our positive frequency-dependent result; however, we demonstrated that mycorrhizal associations were likely not the main mechanism affecting positive frequency dependence. Despite the major limitation of our study, where Topsin-M appears to be killing off pathogens in the soil as well as mycorrhizae, we also found that available soil phosphate was high at the site where our field experiment was planted and where soil for our greenhouse experiment was collected. As mycorrhizae can increase the availability of immobile nutrients, especially soil phosphorus (Bolan 1991), and the level of available phosphorus is considered non-limiting at our site, we predict that another mechanism is likely at play. We suggest that future work should explore alternative mechanisms such as whether plant roots are able to recognize self and non-self (Dudley and File 2007; Mahall and Callaway 1996; Murphy and Dudley 2009).

In summary, we show that invasive genotypes differ in their direction and magnitude of frequency-dependent interactions for different growth measures, and for two key traits we find evidence for positive frequency dependence. For those genotypes that show evidence for positive frequency-dependent interactions, we also eliminate mycorrhizal associations as a factor affecting frequency dependence. Furthermore, we demonstrate that growth measures show differential response to neighbor identity, with some growth measures showing no difference in response regardless of the small-scale frequency neighborhood. We suggest that future work should try to understand what characteristics, both genetic and phenotypic, are associated with positive and negative frequency dependence. In this way, understanding scall-scale genotypic patch structure could better inform the potential for invasion risk.

Acknowledgments This research was supported by US Department of Agriculture (USDA) Hatch and USDA Cooperative Research, Education, and Extension Service Award 2006-03645 (awarded to J.M.). The authors wish to thank Drs Nick Gotelli, Alison Brody, Sara Cahan, Maggie Eppstein, and Don Stratton for their helpful comments on an earlier version of this manuscript. Comments from two anonymous reviewers also greatly improved the manuscript. Colleen 
Armstrong helped care for the plants in the greenhouse and Manisha Patel helped with field work. All experiments comply with current US laws.

\section{References}

Aarssen LW, Turkington R (1983) What is community evolution? Evol Theory 6:211-217

Aarssen LW, Turkington R (1985) Biotic specialization between neighboring genotypes in Lolium perenne and Trifolium repens from a permanent pasture. J Ecol 73:605-614

Agren J (1996) Population size, pollinator limitation, and seed set in the self-incompatible herb Lythrum salicaria. Ecology 77: $1779-1790$

Allee WC (1931) Animal aggregations. University of Chicago Press, Chicago

Antonovics J (1976) The population genetics of mixtures, plant relations in pastures. CSIRO, Melbourne

Antonovics J, Ellstrand NC (1984) Experimental studies of the evolutionary significance of sexual reproduction. 1. A test of the frequency-dependent selection hypothesis. Evolution 38:103-115

Antonovics J, Kareiva P (1988) Frequency-dependent selection and competition: empirical approaches. Philos Trans R Soc Lond B 319:601-613

Bauer CR, Kellogg CH, Bridgham SD, Lamberti GA (2003) Mycorrhizal colonization across hydrologic gradients in restored and reference freshwater wetlands. Wetlands 23:961-968

Beck-Nielsen D, Madsen TV (2001) Occurrence of vesiculararbuscular mycorrhiza in aquatic macrophytes from lakes and streams. Aquat Bot 71:141-148

Bennington CC, Stratton DA (1998) Field tests of density- and frequency-dependent selection in Erigeron annuus (Compositae). Am J Bot 85:540-545

Bever JD (1999) Dynamics within mutualism and the maintenance of diversity: inference from a model of interguild frequency dependence. Ecol Lett 2:52-62

Bever JD (2003) Soil community feedback and the coexistence of competitors: conceptual frameworks and empirical tests. New Phytol 157:465-473

Bolan NS (1991) A Critical-review on the role of mycorrhizal fungi in the uptake of phosphorus by plants. Plant Soil 134:189-207

Bolker BM (2008) Ecological models and data in R. Princeton University Press, NJ

Booth R, Grime JP (2003) Effects of genetic impoverishment on plant community diversity. J Ecol 91:721-730

Broderson C, Lavergne S, Molofsky J (2008) Genetic variation in photosynthetic characteristics among invasive and native populations of reed canarygrass (Phalaris arundinacea). Biol Invasions 10:1317-1325

Callaway RM, Thelen GC, Rodriguez A, Holben WE (2004) Soil biota and exotic plant invasion. Nature 427:731-733

Cooke JC, Lefor MW (1998) The mycorrhizal status of selected plant species from Connecticut wetlands and transition zones. Restor Ecol 6:214-222

Crutsinger GM, Collins MD, Fordyce JA, Gompert Z, Nice CC, Sanders NJ (2006) Plant genotypic diversity predicts community structure and governs an ecosystem process. Science 313: 966-968

Crutsinger GM, Souza L, Sanders NJ (2008) Intraspecific diversity and dominant genotypes resist plant invasions. Ecol Lett 11:16-23

Dudley SA, File AL (2007) Kin recognition in an annual plant. Biol Lett 3:435-438
Elam DR, Ridley CE, Goodell K, Ellstrandt NC (2007) Population size and relatedness affect fitness of a self-incompatible invasive plant. Proc Natl Acad Sci USA 104:549-552

Eppstein MJ, Bever JD, Molofsky J (2006) Spatio-temporal community dynamics induced by frequency dependent interactions. Ecol Modell 197:133-147

Farrer EC, Goldberg DE (2009) Litter drives ecosystem and plant community changes in cattail invasion. Ecol Appl 19:398-412

Futuyma DJ, Wasserman SS (1980) Resource concentration and herbivory in oak forests. Science 210:920-922

Gelman A, Hill J (2007) Data analysis using regression and multilevel/hierarchical models. Cambridge University Press, London

Gilks WR, Thomas A, Spiegelhalter DJ (1994) A language and program for complex Bayesian modelling. Statistician 43:169-177

Gleason HA, Cronquist A (1991) Manual of vascular plants of Northeastern United States and adjacent Canada. The New York Botanical Press, New York

Harper (1977) Plant population biology. Academic, New York

Harpole WS, Suding KN (2007) Frequency-dependence stabilizes competitive interactions among four annual plants. Ecol Lett 10:1164-1169

Hughes AR, Inouye BD, Johnson MTJ, Underwood N, Vellend M (2008) Ecological consequences of genetic diversity. Ecol Lett 11:609-623

Johnson MTJ, Agrawal AA (2005) Plant genotype and environment interact to shape a diverse arthropod community on evening primrose (Oenothera biennis). Ecology 86:874-885

Johnson MTJ, Agrawal AA (2007) Covariation and composition of arthropod species across plant genotypes of evening primrose (Oenothera biennis). Oikos 116:941-956

Johnson MTJ, Lajeunesse MJ, Agrawal AA (2006) Additive and interactive effects of plant genotypic diversity on arthropod communities and plant fitness. Ecol Lett 9:24-34

Kelley S, Clay K (1987) Interspecific competitive interactions and the maintenance of genotypic variation within 2 perennial grasses. Evolution 41:92-103

Kilbride KM, Paveglio FL (1999) Integrated pest management to control reed canarygrass in seasonal wetlands of southwestern Washington. Wildl Soc Bull 27:292-297

Klironomos JN (2002) Feedback with soil biota contributes to plant rarity and invasiveness in communities. Nature 417:67-70

Kulmatiski A, Beard KH, Stevens JR, Cobbold SM (2008) Plant-soil feedbacks: a meta-analytical review. Ecol Lett 11:980-992

Laungani R, Knops JMH (2009) Species-driven changes in nitrogen cycling can provide a mechanism for plant invasions. Proc Natl Acad Sci USA 106:12400-12405

Lavergne S, Molofsky J (2004) Reed canary grass (Phalaris arundinacea) as a biological model in the study of plant invasions. Crit Rev Plant Sci 23:415-429

Lavergne S, Molofsky J (2007) Increased genetic variation and evolutionary potential drive the success of an invasive grass. Proc Natl Acad Sci USA 104:3883-3888

Mahall BE, Callaway RM (1996) Effects of regional origin and genotype on intraspecific root communication in the desert shrub Ambrosia dumosa (Asteraceae). Am J Bot 83:93-98

May RM, Anderson RM (1983) Epidemiology and genetics in the coevolution of parasites and hosts. In: Proc R Soc Lond A 390:219-219

Molofsky J, Bever JD (2002) A novel theory to explain species diversity in landscapes: positive frequency dependence and habitat suitability. In: Proc R Soc Lond B 269:2389-2393

Molofsky J, Bever JD, Antonovics J (2001) Coexistence under positive frequency dependence. In: Proc R Soc Lond B 268:273-277 
Molofsky J, Bever JD, Antonovics J, Newman TJ (2002) Negative frequency dependence and the importance of spatial scale. Ecology 83:21-27

Morrison SL, Molofsky J (1998) Effects of genotypes, soil moisture, and competition on the growth of an invasive grass, Phalaris arundinacea (reed canary grass). Can J Bot-Rev Can Bot 76:1939-1946

Morrison SL, Molofsky J (1999) Environmental and genetic effects on the early survival and growth of the invasive grass Phalaris arundinacea. Can J Bot-Rev Can Bot 77:1447-1453

Murphy GP, Dudley SA (2009) Kin recognition: competition and cooperation in Impatiens (Balsaminaceae). Am J Bot 96:1-7

Neuhauser C, Andow DA, Heimpe GE, May G, Shaw RG, Wagenius S (2003) Community genetics: expanding the synthesis of ecology and genetics. Ecology 84:545-558

Nijjer S, Rogers WE, Siemann E (2007) Negative plant-soil feedbacks may limit persistence of an invasive tree due to rapid accumulation of soil pathogens. In: Proc R Soc Lond B 274:2621-2627

Ostrem L (1988) Studies on genetic variation in reed canary grass Phalaris arundinacea L. III. Seed yield and seed yield components. Hereditas 107:15-168

Qian SS, Shen Z (2007) Ecological applications of multilevel analysis of variance. Ecology 88:2489-2495

Reinhart KO, Callaway RM (2004) Soil biota facilitate exotic Acer invasions in Europe and North America. Ecol Appl 14:1737-1745

Reinhart KO, Callaway RM (2006) Soil biota and invasive plants. New Phytol 170:445-457

Reinhart KO, Packer A, Van der Putten WH, Clay K (2003) Plant-soil biota interactions and spatial distribution of black cherry in its native and invasive ranges. Ecol Lett 6:1046-1050

Rickerl DH, Sancho FO, Ananth S (1992) Vesicular-arbuscular endomycorrhizal colonization of wetland plants. In: Symp Wetl Process Water Qual, vol 23. American Society of Agronomy, Minneapolis, MN, pp 913-916
Ronsheim ML (1996) Evidence against a frequency-dependent advantage for sexual reproduction in Allium vineale. Am Nat 147:718-734

Ronsheim ML, Anderson SE (2001) Population-level specificity in the plant-mycorrhizae association alters intraspecific interactions among neighboring plants. Oecologia 128:77-84

Silvertown J, Charlesworth D (2001) Introduction to plant population biology, 4th edn. Blackwell, Oxford

Stephens PA, Sutherland WJ, Freckleton RP (1999) What is the Allee effect? Oikos 87:185-190

Stevens JT, Beckage B (2009) Fire feedbacks facilitate invasion of pine savannas by Brazilian pepper (Schinus terebinthifolius). New Phytol 184:365-375

Sturtz S, Ligges U, Gelman A (2005) R2WinBUGS: a package for running WinBUGS from R. J Stat Softw 12:1-16

Taylor CM, Hastings A (2005) Allee effects in biological invasions. Ecol Lett 8:895-908

Turkington R (1979) Neighbour relationships in grass-legume communities. IV. Fine scale biotic differentiation. Can J Bot 57:2711-2716

Vellend M, Drummond EBM, Tomimatsu H (2010) Effects of genotype identity and diversity on the invasiveness and invasibility of plant populations. Oecologia 162:371-381

Whitham TG et al (2003) Community and ecosystem genetics: a consequence of the extended phenotype. Ecology 84:559-573

Whitham TG et al (2006) A framework for community and ecosystem genetics: from genes to ecosystems. Nat Rev Genet 7:510-523

Whitlock R, Grime JP, Booth R, Burke T (2007) The role of genotypic diversity in determining grassland community structure under constant environmental conditions. J Ecol 95:895-907

Wilson GWT, Williamson MM (2008) Topsin-M: the new benomyl for mycorrhizal suppression experiments. Mycologia 100:548-554

Wolfe BE, Klironomos JN (2005) Breaking new ground: soil communities and exotic plant invasion. Bioscience 55:477-487 